\title{
Cyto-Fluid Dynamic Theory of Atomization Processes
}

\author{
K. Naitoh ${ }^{1}$ \\ 1 Nissan Motor Co. Ltd, Engine and Powertrain Research Laboratory, Natsushima-cho, Yokosuka-shi, Kanagawa 237 - Japan \\ e-mail: k-naito@mail.nissan.co.jp
}

\begin{abstract}
Résumé - Une théorie cyto-fluide des processus d'atomisation - Cet article propose une théorie générale de l'atomisation des sprays. À partir de cette théorie, des modèles déjà décrits, empiriques ou basés sur des analogies peuvent être obtenus et leurs constantes arbitraires déterminées. De plus, cette théorie fournit une formulation détaillée du phénomène de rupture succédant à des collisions de gouttes. Enfin, il est montré sur la base de cette théorie que le phénomène d'atomisation du carburant est équivalent mathématiquement à une prolifération cellulaire. La recherche intégrée dans les domaines de l'atomisation des liquides et la division cellulaire nous apportera des solutions aux impasses de chacun de ces deux domaines, puisque le but commun de ces deux champs de recherche est une atomisation plus rapide de gouttes d'hydrocarbure.
\end{abstract}

Mots-clés : cyto-fluide, atomisation, spray.

\begin{abstract}
Cyto-Fluid Dynamic Theory of Atomization Processes - A universal theory of spray atomization processes is proposed. Based on the proposed theory, previously reported empirical models and analogy models such as the TAB model and the OPT (oval-parabola trajectory) model can be derived along with their arbitrary constants. Moreover, this theory provides a detailed formulation for breakup phenomena after two droplets collide. Finally, it is shown on the basis of this theory that the atomization processes of fuel coming from ancient living systems are similar to cell proliferation mathematically. The integrated research including liquid atomizations and actual biological cell divisions will bring us ways out of impasses for each field, since the target of both research fields is faster breakup of hydrocarbon droplets.
\end{abstract}

Keywords: Cyto-Fluid, atomiszation, spray.

\section{INTRODUCTION}

Models of spray atomization processes in an automotive engine have so far been limited mostly to empirical equations $[1,2]$ or analogy models $[3,4]$, which either have a limited range of application or are imprecise. This paper proposes a highly universal dynamic theory that allows the arbitrary constants of previously proposed empirical equations and analogy models to be derived uniformly on the basis of the first principle of fluid dynamics. This theory also makes it possible to derive a model of more complex atomization phenomena such as droplet breakup following the collision of two fuel droplets, which previously reported models have not been able to treat. Moreover, it is also shown that the shape of fuel droplets can be found by using the present theory. Based on this theory, a fuel injector has been designed for promoting spray atomization at relatively low fuel injection pressures. Lastly, it is shown on the basis of the proposed dynamic theory that fuel atomization processes are mathematically analogous to cell proliferation, which suggests possibilities for synergistic research between the fields of fluid dynamics and life sciences.

\section{CYTO-FLUID DYNAMIC THEORY FOR ATOMIZATION PROCESSES $[12,13]$}

It can be said that a particle method has the advantage of making it easy to grasp the essence of a phenomenon. Therefore, in this paper we will approximate and analyze the liquid phase portion of the atomization processes as a mass of spheroidal liquid particles, rather than analyzing the twophase flow field with partial differential equations.

First, the following assumptions are introduced.

- The droplet shape is assumed to be a spheroid, whose longto-short-axis ratio changes with time. Letting $a(t)$ and $b(t)$ denote one-half of the length of the long axis and short axis, 


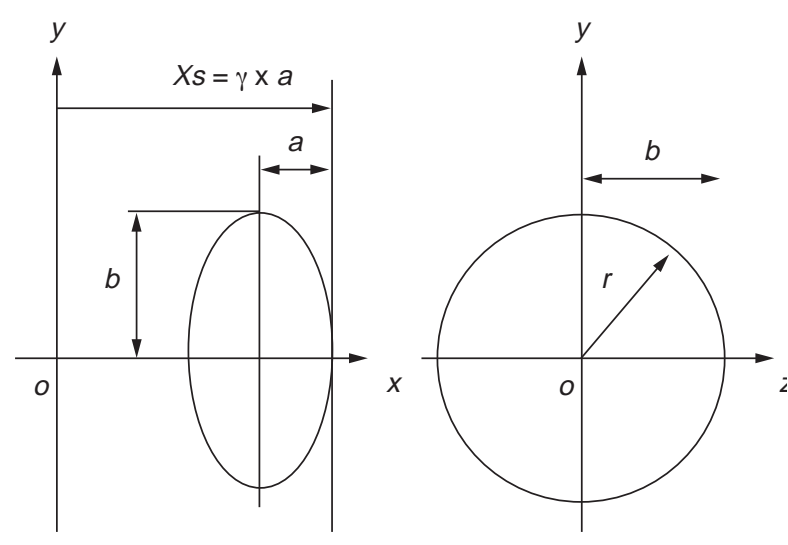

Figure 1

Positional relation of the two diameters and origin of a spheroidal fuel droplet.

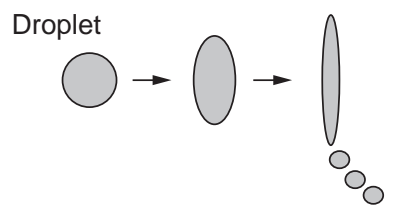

(a) Breakup of droplet flying in the air

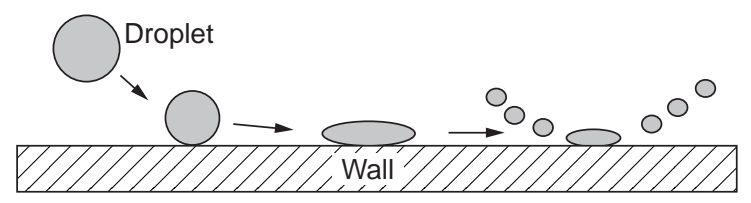

(b) Wall impingement of droplet

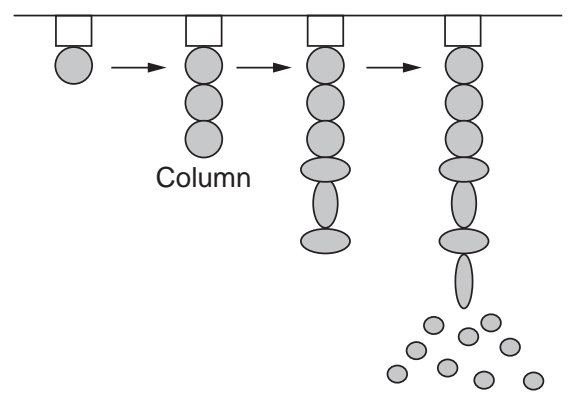

(c) Breakup of liquid column

Figure 2

Breakup of droplet flying in the air, wall-impingement of droplets, and breakup of liquid column jet.

respectively, the radius $r_{d}$ of a droplet when it is a spherical globule can be given by $r_{d}^{3}=a b^{2}$ (Fig. 1, Fig. 2a, Fig. 2b).

- In the breakup of the liquid column, droplets having the same diameter as that of the column are assumed to form a mass in which any number of droplets are in contact in a straight line (Fig. 2c). The individual droplets forming this mass have the above-mentioned spheroidal shape and undergo oscillation and deformation.

- The motions of free droplets and connecting droplets in liquid column jets consist of translation motion of their center of gravity, rotational motion around their center of gravity and deformation motion (internal motion) under a fixed center of gravity. Here, we ignore rotational motion around the center of gravity and derive basic approximation equations describing the temporal change in the shape deformation of droplets in relation to translation motion.

- The internal flow of each droplet is potential flow.

- The temporal change in the shape of each droplet is determined by accelerated motion of the droplet center of gravity, droplet inner inertia, surface tension and the resistance given by the surrounding air. (When droplets are in contact in a line, accelerated motion of the center of gravity of adjacent droplets affects the oscillation and deformation of the droplet of interest).

Transforming and approximating the partial differential equation for potential flow on the basis of these assumptions yields Equation (1) below [6]. This ordinary differential equation is used to find the temporal change in the short-tolong-axis ratio $\gamma(=a / b)$, i.e., the deformation ratio, of the spheroid in Figure 1. The four terms on the right-hand side are the inertia of internal flow, surface tension, accelerated motion of droplet surface position $\left(\gamma_{s}\right)$, and the resistance given by the surrounding air.

$$
\begin{gathered}
\frac{\mathrm{d}^{2} \gamma}{\mathrm{d} \bar{t}^{2}}=\frac{1}{3 \gamma} \frac{\gamma^{2}-2}{\gamma^{2}-1 / 2}\left(\frac{\mathrm{d} \gamma}{\mathrm{d} \bar{t}}\right)^{2}+\frac{3}{8} \frac{2 \gamma^{2}-1 / \gamma-\gamma}{\gamma^{2}-1 / 2} \gamma \\
+3 \frac{\gamma^{7 / 3}}{\gamma^{2}-1 / 2}\left(\frac{\mathrm{d}^{2} \gamma_{s}}{\mathrm{~d} \bar{t}^{2}}\right)-\frac{3}{8} W_{e 2} \cdot C_{d} \cdot \frac{\gamma^{5 / 3}}{\gamma^{2}-1 / 2} \\
\bar{t}=\varpi t, \varpi^{2}=8 \sigma /\left(\rho_{L} r_{d}{ }^{3}\right), C_{d}=\Delta P /\left[\rho_{a}\left(U_{d}-U_{a}\right)^{2}\right], \\
W_{e 2}=\rho_{a} \frac{\left(U_{d}-U_{a}\right)^{2} r_{d}}{\sigma}
\end{gathered}
$$

where:

$\Delta P$ pressure difference fore and aft of a droplet

$U_{a}$ air velocity

$U_{d}$ particle velocity

$\sigma$ surface tension coefficient

$\rho_{a}$ air density

$\rho_{L}$ liquid density.

This fundamental equation provides a uniform description of deformation motion in a variety of cases. The following discussion will show that various empirical equations and analogy models can be derived on the basis of this expression and that their arbitrary constants can also be determined. 


\subsection{Droplet Breakup in the Air (Fig. 2a)}

For droplets suspended in the air, the relation between the droplet surface position $\gamma_{s}$ and the droplet deformation ratio $\gamma$ is given by:

$$
\frac{\mathrm{d}^{2} \gamma_{s}}{\mathrm{~d} \bar{t}^{2}}=6 \gamma^{3} \frac{\mathrm{d}^{2} \gamma}{\mathrm{d} \bar{t}^{2}}-2 \gamma^{2}\left(\frac{\mathrm{d} \gamma}{\mathrm{d} \bar{t}}\right)^{2}
$$

Accordingly, substituting this expression into Equation (1) ultimately yields

$$
\begin{aligned}
\frac{\mathrm{d}^{2} \gamma}{\mathrm{d} \bar{t}^{2}}= & \frac{1}{3 \gamma} \frac{\gamma^{2}+2}{\gamma^{2}+1 / 2}\left(\frac{\mathrm{d} \gamma}{\mathrm{d} \bar{t}}\right)^{2}-\frac{3}{8} \frac{2 \gamma^{2}-1 / \gamma-\gamma}{\gamma^{2}+1 / 2} \gamma \\
& +\frac{3}{8} W_{e 2} \cdot C_{d} \cdot \frac{\gamma^{5 / 3}}{\gamma^{2}+1 / 2}
\end{aligned}
$$

The denominators of this equation always have positive values. Additionally, it is also seen that surface tension acts as a stabilizing force for all the values of $\gamma$.

Next, we will examine droplet behavior in the vicinity of $\gamma=1$. By letting $\gamma=1+\delta \gamma(\delta<<1)$ and substituting it into Equation (2), the infinitesimal amounts are eliminated. In order to make a comparison with the equation of motion of the TAB model [3], we will replace $\delta \gamma$ with $\left(y=\gamma_{-1}\right)$, which results in:

$$
\begin{aligned}
& \frac{\mathrm{d}^{2} \gamma}{\mathrm{d} t^{2}}=\frac{2}{3}\left(\frac{\mathrm{d} y}{\mathrm{~d} t}\right)^{2}-8 K_{x} \cdot y+0.848 \cdot K_{x} \cdot W_{e 2} \\
& K_{x}=\sigma /\left(\rho_{L} r_{d}{ }^{3}\right)
\end{aligned}
$$

The nonlinear equation Equation (3a) has an analytical solution [4].

Removing the first term (inner inertia) from this equation gives the TAB model shown in Equation (3b). It is seen that the constants of the equation above are nearly identical to those of the TAB model.

$$
\frac{\mathrm{d}^{2} y}{\mathrm{~d} t^{2}}=-8 K_{x} \cdot \gamma+0.667 \cdot K_{x} \cdot W_{e 2}
$$

where $t$ is time. It should be stressed that the TAB model can not be used for extremely high Weber number conditions, since Equation (3a) is derived for small deformations.

By letting $\gamma=8$ and substituting it into Equation (2), the infinitesimal amounts are eliminated. Then, we get:

$$
\frac{\mathrm{d}^{2} \gamma}{\mathrm{d} t^{2}}=-6 K_{x} \cdot y+1.272 \cdot K_{x} \cdot W_{e 2} \cdot|y|^{-1 / 3}
$$

The second term on the right-hand side of Equation (3c) is weakened by $|\gamma|^{-1 / 3}$ at large deformations (large Weber numbers). This modified formulation leads to the accurate prediction of child droplet sizes for large deformations.

\subsection{Droplet Breakup Due to Wall-Impingement (Fig. 2b) and Breakup of Equal-Sized Droplets Connecting}

In this case, the droplet surface position $\gamma_{\mathrm{s}}$ is given by:

$$
\frac{\mathrm{d}^{2} \gamma_{s}}{\mathrm{~d} \bar{t}^{2}}=0
$$

and substituting this expression into Equation (1) produces:

$$
\begin{aligned}
\frac{\mathrm{d}^{2} \gamma}{\mathrm{d} \bar{t}^{2}}= & \frac{1}{3 \gamma} \frac{\gamma^{2}-2}{\gamma^{2}-1 / 2}\left(\frac{\mathrm{d} \gamma}{\mathrm{d} \bar{t}}\right)^{2}+\frac{3}{8} \frac{2 \gamma^{2}-1 / \gamma-\gamma}{\gamma^{2}-1 / 2} \gamma \\
& -\frac{3}{8} W_{e 2} \cdot C_{d} \cdot \frac{\gamma^{5 / 3}}{\gamma^{2}-1 / 2}
\end{aligned}
$$

This equation holds true by removing the singular point of $\gamma=1 / \sqrt{2}$ that makes the denominator of each term zero. (This singularity will be discussed in a later section.) In this case, surface tension acts as either a stabilizing force or a destabilizing force depending on the value of $\gamma$. When the infinitesimal displacement of a droplet is taken into account, the equation can also be written as:

$$
\frac{\mathrm{d}^{2} \gamma}{\mathrm{d} t^{2}}=-C_{1}\left(\frac{\mathrm{d} y}{\mathrm{~d} \bar{t}}\right)^{2}-C_{2} K_{x} \cdot \gamma
$$

where $C_{1}$ and $C_{2}$ denote the arbitrary constants, which can be determined from Equation (4). Equation (5) is identical with the oval-parabola trajectory (OPT) model [4], an analogical model for wall-impingement of liquid droplets. Equation (5) also has an analytical solution [4].

Equation (5) models also the collision process of equalsized droplets, because Equation (5) for wall-impingement is developed with the assumption of a virtual equal-sized droplet inside wall.

In the case of Equation (5), the maximum deformation amount $y_{\max }$ at $\mathrm{d} y / \mathrm{d} t=0$ has two different values on account of the nonlinearity of the first term on the right-hand side [4]. With the TAB model [3], $y_{\max }$ has only one value because of linear simple oscillation. Since droplets produced in the breakup process are nearly proportional in size to the maximum amount of deformation, it is thought that the existence of these two maximum deformation values gives rise to a bimodal droplet size distribution. Figure 3 shows the bimodal frequency distribution plotted against the child droplet size, when the single-sized parent droplets with a diameter of $160 \mu \mathrm{m}$ and a droplet speed of $12 \mathrm{~m} / \mathrm{s}$ hit a solid wall at an impingement angle of 30 degrees [4]. The formulation with Equation (5) and the energy conservation 

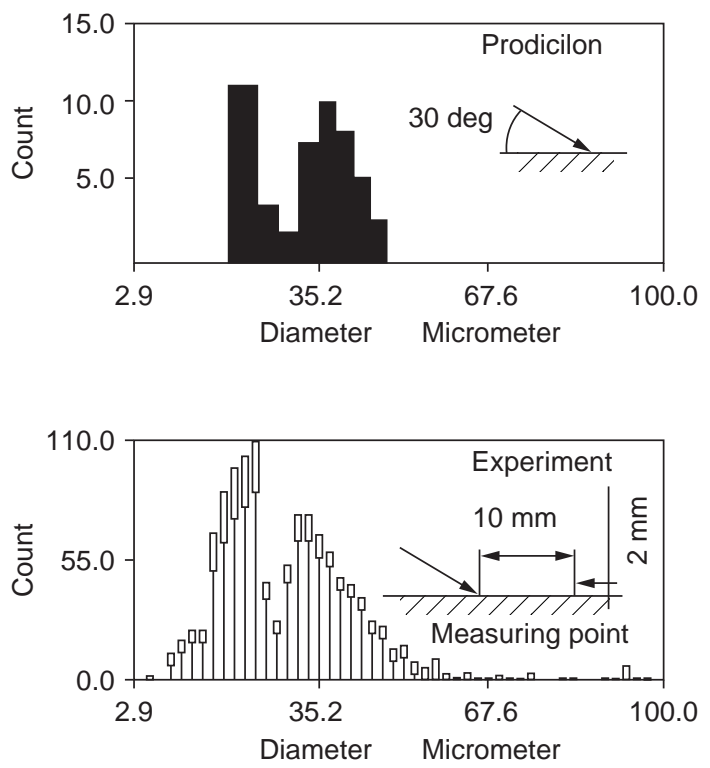

Figure 3

Predicted frequency distribution plotted against the child droplet size and the corresponding experimental data. (Parent droplet size $=160 \mu$, impingement speed $=12.0 \mathrm{~m} / \mathrm{s}$, impingement angle $=30$ degrees).

law can predict also the mass of fuel remaining on the solid wall after droplet impingement for a wide range of parent droplet sizes and impingement speeds.

\subsection{Breakup of Liquid Column Jet (Fig. 2c) and Connection of Two Different-Size Droplets}

In this section we will consider the process from the moment the fuel jet, having a certain cross-sectional area, leaves the fuel injector until it breaks up. The essential pattern for this process is a system represented by the oscillation of two droplets in a state of contact. (Actually, we can observe that a column jet breaks up after forming droplets in contact in a straight line at a low Weber number.) The equation of motion for the deformation motion of droplets 1 and 2 is expressed as follows:

$$
\begin{aligned}
& A(\vec{\gamma}) \frac{\mathrm{d}^{2} \vec{\gamma}}{\mathrm{d} \bar{t}^{2}}=B(\vec{\gamma})\left(\frac{\mathrm{d} \vec{\gamma}}{\mathrm{d} \bar{t}}\right)\left(\frac{\mathrm{d} \vec{\gamma}}{\mathrm{d} \bar{t}}\right)^{T}+C(\vec{\gamma}) \vec{\gamma}-D\left(\vec{\gamma}, W_{e 2}\right) \\
& \vec{\gamma}=\left[\begin{array}{l}
\gamma_{1} \\
\gamma_{2}
\end{array}\right]
\end{aligned}
$$

where $\gamma_{1}$ and $\gamma_{2}$ denote the deformations of two droplets.

Moreover, the oscillation of droplets 1 and 2 can be expressed approximately by Equations (7a) and (7b).

Droplet 1:

$$
\begin{aligned}
\frac{\mathrm{d}^{2} \gamma_{1}}{\mathrm{~d} \bar{t}^{2}}= & \frac{1}{3 \gamma_{1}} \frac{\gamma_{1}{ }^{2}-2}{\gamma_{1}{ }^{2}-1 / 2}\left(\frac{\mathrm{d} \gamma_{1}}{\mathrm{~d} \bar{t}}\right)^{2} \\
& +\frac{3}{8} \frac{2 \gamma_{1}{ }^{2}-1 / \gamma_{1}-\gamma_{1}}{\gamma_{1}{ }^{2}-1 / 2} \gamma_{1} \\
& -\frac{3}{8} W_{e 2} \cdot C_{d} \cdot \frac{\gamma_{1}{ }^{5 / 3}}{{\gamma_{1}{ }^{2}-1 / 2}^{2}}
\end{aligned}
$$

Droplet 2:

$$
\begin{aligned}
\frac{\mathrm{d}^{2} \gamma_{2}}{\mathrm{~d} \bar{t}^{2}}= & \frac{1}{3 \gamma_{2}} \frac{\gamma_{2}^{2}+2}{\gamma_{2}^{2}+1 / 2}\left(\frac{\mathrm{d} \gamma_{2}}{\mathrm{~d} \bar{t}}\right)^{2} \\
& -\frac{3}{8} \frac{2 \gamma_{2}{ }^{2}-1 / \gamma_{2}-\gamma_{2}}{\gamma_{2}{ }^{2}+1 / 2} \gamma_{2} \\
& +\frac{3}{8} W_{e 2} \cdot C_{d} \cdot \frac{\gamma_{2}{ }^{5 / 3}}{\gamma_{2}{ }^{2}+1 / 2}
\end{aligned}
$$

Equation (7a) for the oscillation of droplet 1 is identical to Equation (4), and surface tension acts primarily as a destabilizing force. Equation (7b) for the oscillation of droplet 2 is identical to Equation (2), and surface tension acts as a stabilizing force. Since the actual breakup is thought to be caused by the droplet that is more unstable, the third term of Equation (7b) becomes the controlling rate at $W_{e}=\infty$; deleting the nonlinear term in the vicinity of $\gamma=1$ allows the following approximation to be made:

$$
\frac{\mathrm{d}^{2} \gamma_{2}}{\mathrm{~d} \bar{t}^{2}}=-\frac{3}{4} \gamma_{2}+\frac{1}{4} \cdot W_{e 2} \cdot C_{d}
$$

Therefore, from this equation the well-known breakup time can be derived as follows:

$$
t_{d}=C_{z z} \sqrt{\frac{\rho_{L}}{\rho_{a}}} \frac{r_{d}}{\left|U_{d}-U_{a}\right|}
$$

At $W_{e}=0$, the second term of Equation (7a) becomes the controlling rate; the following expression is obtained in the vicinity of $\gamma=1$ :

$$
\frac{\mathrm{d}^{2} \gamma_{1}}{\mathrm{~d} \bar{t}^{2}}=-\frac{3}{4} \gamma_{1}
$$

and the well-known breakup time is derived as:

$$
t_{d}=C_{z} \sqrt{\frac{\rho_{L} r_{d}^{3}}{8 \sigma}}
$$

The foregoing discussion indicates that the droplet diameter and number after various patterns of breakup can be found from Equation (1) or an equation of motion that approximates it, by combining the laws concerning the conservation of energy and the conservation of mass, 
provided that the extent of the deformation, i.e., the thickness at the moment of maximum deformation, is known. Further details are given in references [3, 4, 6, 12, 13].

This theoretical approach also makes it possible to calculate the spray formation processes of hollow-cone injectors for direct-injection gasoline engines (Fig. 4) [6].

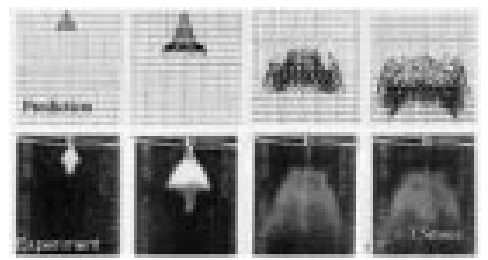

(a) Initial cone angle $=60$ degree, back pressure $=0.1 \mathrm{Mpa}$

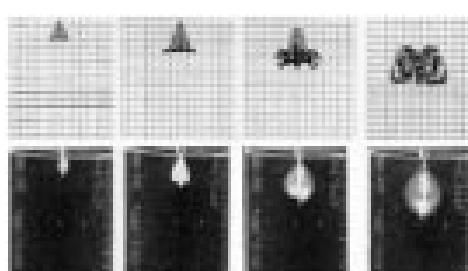

(b) Initial cone angle $=60$ degree, back pressure $=1.1 \mathrm{Mpa}$

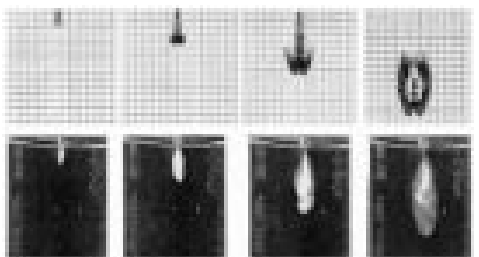

(c) Initial cone angle $=20$ degree, back pressure $=1.1 \mathrm{Mpa}$

Figure 4

Predicted spatial distribution of fuel spray droplets injected in a constant volume and the corresponding experimental visualizations.

\section{$2 \sqrt{2}$ PHENOMENA IN LIQUID ATOMIZATION PROCESSES}

In Equation (4), the fact that the denominators become zero at $\gamma=1 / \sqrt{2}$ does not mean the theory proposed here cannot be used. In actuality, when Equation (4) is applied, the approximation of $\mathrm{d} \gamma / \mathrm{d} t=0$ is allowed at $\gamma=1 / \sqrt{2}$ [6]. The significant point of this theory is that it indicates a spheroid cannot be maintained at $\gamma=1 / \sqrt{2}$. It is known from experimental results that droplets are not actually spheroids at $\gamma=1 / \sqrt{2}$, but rather they have a shape resembling that of a brimmed hat or a flying saucer [7, 11]. In other words, what is significant is that a model which posits a spheroidal shape is in itself valid as a first-order approximation of a droplet, and it also permits a further discussion of the droplet shape. The framework of the particle model described in Section 2 can also be extended to a variety of different modal shapes, such as that of confetti candies, in addition to a spheroid.
Figure 5 shows the predicted time-dependent droplet deformations, after two different-sized droplets collide. (The values $X 1\left(=1-\gamma_{1}\right)$ and $X 2\left(=1-\gamma_{2}\right)$ imply the deformations of droplets 1 and 2.) Calculations are made with Equation (6) [4]. We can see that atomization is best promoted when the ratio of the diameters of two droplets is on the order of $1: \sqrt{2}$. Based on that insight, a prototype fuel injector was fabricated which is constructed so that the fuel jets discharged from two different-sized nozzle holes collide following injection (Fig. 6).

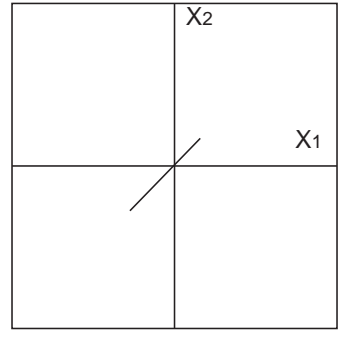

(a) 100:100 micron

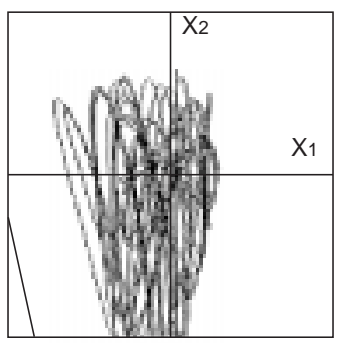

(c) 100:150 micron

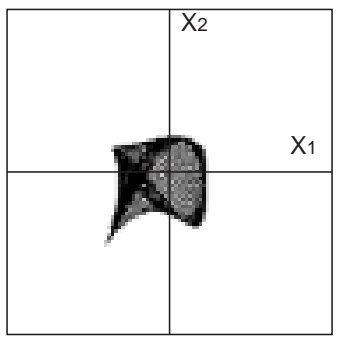

(b) 100:130 micron

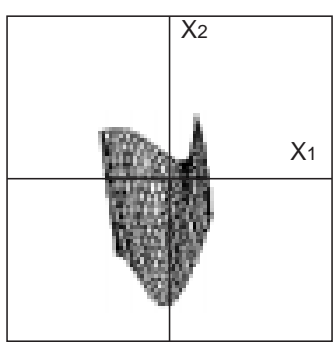

(b) 100:200 micron
Figure 5

Calculated time-histories of the extent of droplet deformation following the collision of two different-sized droplets.

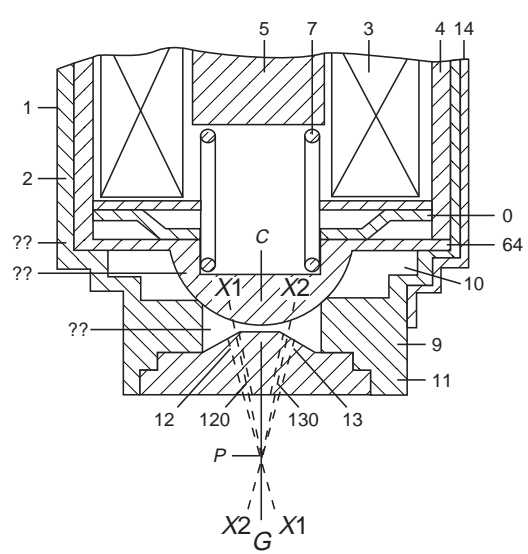

Figure 6

Prototype injector having two different-sized nozzle holes. (Two liquid columns injected from the nozzle holes collide each other before breakup). 


\section{SIMILARITY BETWEEN CELL PROLIFERATION AND LIQUID DROPLET ATOMIZATION}

\subsection{Actual Biological Cell with Flying Saucer Shape $[12,13]$}

Artificial cells and actual living cells also show this type of flying-saucer-like shape in the vicinity of $\gamma=1 / \sqrt{2}$ [8].

This flying-saucer-like shape of living cells is natural, since actual biological cells are occupied with water. However, it should be stressed that objects such as microtubules in biological cells are also important for cell shapes and division processes.

\subsection{Size Ratio of Child Cells [12, 13]}

It is also well known that when a cell divides the size ratio of two resulting child cells is nearly equal to $1: \sqrt{2}$ [10]. In other words, it is possible that cell proliferation also makes use of instabilities of a fluid dynamics nature.

We are comfortable in seeing the objects with the $1: \sqrt{2}$ size ratio, which are the papers of $\mathrm{B} 5, \mathrm{~A} 4$, and $\mathrm{A} 3$ sizes and the golden section in painting arts. This may be related to the connections between neural "cells" in our brains.

\subsection{Cell Cycle $[12,13]$}

It is well known that the period necessary for the division of one ovum fertilized (first cell cycle) is longer than that of the division of two interacting cells (second cell cycle). The present theoretical model may predict the difference between first and second cell cycles.

The cells during first and second cell cycles are dominated by Equations. (2) and (4), respectively. This is because two interacting cells during second cell cycle are similar to two connecting droplets, although the single cell during first cell cycle is similar to single free droplet. Then the surface tension can become an instability effect for connec-ting cells. Thus the second cell cycle is shorter than that of first cell cycle.

However, the present hypothesis must be examined further, because the first cell cycle may include a delay time before the mother cell, ovum fertilized, is mature.

\section{CONCLUSION}

The above results suggest that it is possible to regard the three forces in cells (protoplasm flow, cell membrane tension and pressure difference due to energy supply by ATP) as being mathematically equivalent to the inner inertia, surface tension and momentum of liquid droplets, respectively. This indicates that the dynamics discussed here are not simply limited to those of two-phase flow, rather they might also form the foundation of the kinetics (flexible kinetics) of living organisms.

By drawing upon this theory to conduct integrated research on nonliving fuel atomization processes and living cell kinetic phenomena, it is possible that it might show ways out of impasses in each field.

\section{REFERENCES}

1 Senda, J. et al. (1994) Modeling of Diesel Spray Impingement on a Flat Wall. SAE Paper No. 941894.

2 Hiroyasu, H. et al. (1974) Fuel Droplet Size Distribution in Diesel Combustion Chamber. SAE Paper No. 740715.

3 O'Rourke, P.J. and Amsden, A.A. (1987) The TAB Method for Numerical Calculation of Spray Droplet Breakup. SAE Paper No. 872089.

4 Naitoh, K. et al. (1994) Numerical Predeiction of Fuel Secondary Atomization Behavior in SI Engine Based on the Oval-Palabola Trajectories Model. Trans. of SAE Paper No. 940526.

5 Reitz, R.D. and Bracco, F.V. (1982) Mechanisms of Atomization of a Liquid Jet. Physics of Fluids, 25, 10.

6 Naitoh, K. et al. (1996) Synthesized Spheroid Particle Method for Calculating Spray Phenomena in Direct-Injection SI Engines. Trans. of SAE Paper No. 962017.

7 Chandra, S. and Avedisian, C.T. (1991) Proc. R. Soc., London A.

8 Houtani, K. (1995) Biohistory, 3, 2.

9 Naitoh, K. (1996) United States Patent.

10 Homma, K. et al. (1989) Cell Growth Kinetics. J. of Cell Science, 92.

11 Umemura, A. (1990) Proceedings of Tsukuba International Workshop on Mechanics of Reacting Flows.

12 Naitoh, K. (1998) Applications of Cytofluid Dynamic Theory to Engineering Problems. Proceedings of Japan Society of Industrial and Applied Mathematics.

13 K. Naitoh (1998) Proceedings of Comodia 98.

Final manuscript received in March 1999 